\title{
Aportación científica de la medicina interna hospitalaria gallega a congresos nacionales e internacionales de su especialidad
}

\author{
J. MONTES SANTIAGO, R. PÉREZ ÁLVAREZ \\ Servicio de Medicina Interna. Hospital Meixoeiro. Vigo
}

\begin{abstract}
CONTRIBUTION OF GALICIAN HOSPITALS TO NATIONAL AND INTERNATIONAL INTERNAL MEDICINE SCIENTIFIC MEETINGS
\end{abstract}

\section{RESUMEN}

Objetivos: Cuantificar las comunicaciones de la medicina interna hospitalaria gallega a congresos nacionales e internacionales de su especialidad y describir sus campos preferentes de investigación clínica.

Material y método: Tabulación de las comunicaciones de hospitales gallegos tras examinar los Libros de comunicaciones de los 4 últimos Congresos Nacionales de Medicina Interna (1994-2000) y su comparación con el de hace una década (1984); de los 2 últimos europeos (1999 y 2001) y último mundial (2000). Se excluyeron comunicaciones referentes a sólo uno o dos casos.

Resultados: Se objetivó una paulatina y continuada incorporación de hospitales a estas comunicaciones (mínimo: 2 (1984), máximo: 12 (1996), con un promedio de 5 ó 6). La cifra de comunicaciones se ha mantenido y estabilizado en torno al $5 \%$ de las nacionales. Son predominantes la investigación cardiovascular y en enfermedades infecciosasSIDA.

Conclusiones: Se observa una creciente incorporación de los internistas hospitalarios gallegos a la investigación clínica comunicada en congresos nacionales e internacionales. Dicha producción ha alcanzado un elevado grado de continuidad sobre todo en los campos de investigación cardiovascular e infecciosas-SIDA.

PALABRAS CLAVE: Medicina Interna. Investigación clínica hospitalaria.

\section{ABSTRACT}

Objectives: To evaluate the scientific contributions of internal medi cine services from Galicia to nationals and internationals specialty mee tings and to describe theirs preferences in clinical research..

Material and method: We analysed the communications from the Abstracts books of the last four Spanish Internal Medicine National Con gress (1994-2000 period) in comparison with that of 1984, the last two European Congress (1999 and 2001 ) and the last World Congress (2000). Case reports were excluded.

Results: An increasing number of communicating hospitals and a sustained number of reports was observed with a minimum of 2 hospi tals (1984) to a maximum of 12 (1996) with 5-6 hospitals on average. Number of communications became stable and reached $5 \%$ of the whole spanish reports. Cardiovascular and AIDS-Infectious diseases were the preferred research fields.

Conclusions: An increment of scientific communications from a gro wing number of galician hospitals was observed. This scientific produc tion became continuous and stabilized notoriously on cardiovascular and AIDS-infectious disease fields.

KEY WORDS: Internal Medicine. Clinical investigation.

Montes Santiago J. Pérez Álvarez R. Aportación científica de la medicina interna hospitalaria gallega a congresos nacionales e internacionales de su especialidad. An Med Interna (Madrid) 2002; 19: 567-570.

\section{INTRODUCCIÓN}

En febrero de 1952 quedaba constituida la Sociedad Española de Medicina Interna (SEMI), promovida entre otros por los doctores Jiménez Díaz, Marañón y Pedro Pons, siendo su primer presidente el Dr. D. Carlos Jiménez Díaz. Se cumple por tanto este año el 50 aniversario de la fundación de dicha sociedad. Paulatinamente, y como filiales de esta "sociedad madre" se fueron creando y adhiriendo diversas sociedades regionales. Con ello se facilitaba el cumplimiento de uno de los estatutos fundacionales de la SEMI que estipulaba que en los años pares se celebraría el Congreso Nacional en una ciudad española y en los impares una Reunión en Madrid (1).

A pesar de este antecedente, no sería hasta 31 años más tarde cuando se constituyera la Sociedad Gallega de Medicina Interna (SOGAMI). Ello fue posible a instancias principalmente de los jefes de los Servicios de Medicina Interna de los hospitales del INSALUD, radicados en las principales ciudades gallegas. Se eligió como primer presidente al profesor M. Noya García de Santiago de Compostela. A partir de ese momento la celebración itinerante anual en las diversas ciudades gallegas de las Reuniones de la SOGAMI y el acuerdo

Trabajo aceptado: 25 de julio de 2002

Correspondencia: Julio Montes Santiago. Servicio de Medicina Interna. Hospital Meixoeiro. 36200 Vigo (Pontevedra). e-mail: julio.montes.santiago@ sergas.es 
alcanzado para la reconversión de la revista Galicia Clínica (fundada en 1929) como "órgano oficioso" de la SOGAMI (2), permitieron un contacto relativamente fluido entre los diversos internistas gallegos.

A pesar del momento de esplendor que para la medicina gallega había supuesto la figura de Roberto Novoa Santos (3) -fallecido prematuramente en 1933 y tan admirado por Marañón- y su magisterio en España y América a través de las sucesivas ediciones de su célebre Patología general, es preciso reconocer que la situación periférica y las pésimas comunicaciones habían motivado que la Medicina Interna de Galicia, salvo excepciones individuales, viviera un tanto aislada de la del resto de España. Esto es claro, al menos, en lo referente a la participación en las actividades de la SEMI como muestra, por ejemplo, la ya mencionada fundación tardía de la SOGAMI o el hecho de que al $16^{\circ}$ Congreso Nacional de la SEMI de 1984 celebrado en Salamanca -ciudad relativamente cercana y accesible desde Galicia-, sólo se presentaran 3 comunicaciones de 2 hospitales gallegos (4). Sin embargo, sólo 12 años después y como el Dr. Gil Extremera, anterior presidente de la SEMI ha reconocido, la celebración en 1996 del Congreso Nacional en Santiago de Compostela supuso un punto de inflexión en los congresos nacionales de la SEMI, tanto por la alta participación como por la calidad de ponencias presentadas (1). Se certificaba así de manera definitiva la total incorporación de la medicina interna gallega a los quehaceres de la SEMI.

El objetivo de este trabajo, sin precedentes previos que conozcamos, es describir cuantitativamente las aportaciones científicas de la Medicina Interna hospitalaria gallega a los 4 últimos Congresos Nacionales de Medicina Interna, comparándolos con el decenio previo, así como su aportación a los 2 últimos Congresos Europeos y último Mundial. También se estudian los campos preferentes de la actividad clínica investigadora de los internistas hospitalarios de esta Comunidad.

\section{MATERIAL Y MÉTODOS}

Se examinaron los Libros de comunicaciones de los Congresos Nacionales de Medicina Interna de los últimos 6 años: $21^{\circ}$ Congreso: Alicante 1994 (presidente: Prof. J. Merino Sánchez), coincidente también con el Congreso de la European Association of Internal Medicine (EAIM); $22^{\circ}$ Congreso: Santiago de Compostela 1996 (presidente Prof. J.M. Martínez Vázquez); $23^{\circ}$ Congreso: Granada 1998 (presidente: Prof. B. Gil Extremera); $24^{\circ}$ Congreso: Zaragoza 2000 (presidente: Prof P. Cía Gómez) (5-8). Como punto comparativo se examinó también el Libro de comunicaciones del $16^{\circ}$ Congreso Nacional: Salamanca 1984 (presidente: Prof. Sisinio De Castro del Pozo) (4). Fueron revisados también los Libros de resúmenes del $2^{\circ}$ Congreso de la European Federation of Internal Medicine (EFIM): Florencia, Italia, 1999 (presidente: F. Dammacco) (9), $3^{\circ}$ Congreso de la EFIM: Edimburgo, Reino Unido, 2001 (presidente: C. Davidson) (10) y del $25^{\circ}$ World Congress of Internal Medicine: Cancún, México, 2000 (presidente: A. Lifshitz) (11). Se registraron las comunicaciones procedentes de todos los hospitales gallegos. En los casos de publicaciones multihospitalarias se adscribió la comunicación al hospital del primer firmante. Para la elaboración de la tabla 2 se redistribuyeron bajo otros contenidos algunas de las comunicaciones de difícil adscripción, y recogidas fundamen- talmente bajo el epígrafe de "miscelánea" u "otros temas". Por ejemplo, la adscripción de "neumonías en pacientes ancianos" bajo el epígrafe de "Geriatría" fue recolocada bajo el grupo de "Infecciosas". Se excluyeron del análisis en el presente estudio, y tampoco se contabilizaron en el recuento general de todos los congresos, todas aquellas comunicaciones presentadas bajo el formato de "Comunicaciones a propósito de uno o dos casos".

\section{RESULTADOS}

Se exponen en la tabla I. Si exceptuamos el congreso Santiago-96 en que se produjo un notable aumento del número y procedencia de las comunicaciones debido al factor de proximidad (las comunicaciones gallegas procedentes de 12 hospitales llegaron a representar el $16 \%$ del total de comunicaciones), en el resto de congresos se ha llegado a una cifra de comunicaciones estables que sobrepasan el 5\% de las comunicaciones españolas. Se observan ocasionales aumentos como el 14\% del congreso Florencia-99, pero esto se hizo a expensas de un sólo hospital (Universitario de Santiago). Lo más importante es constatar que, por ejemplo, en el congreso Granada-98 las comunicaciones procedieron de 8 hospitales, superando así ampliamente los únicos 2 hospitales de 1984. Esta remisión de comunicaciones por parte de casi todos los hospitales gallegos ha continuado, resultando así un promedio de 5 ó 6 los que mandan una comunicación a cualquier congreso-, con la excepción de los hospitales comarcales de más reciente creación. Algunos de estos comienzan ya a remitir comunicaciones a congresos europeos (Hospital del Barbanza: 1 comunicación al congreso Edimburgo-01) (12). No obstante, se advierte que hospitales muy activos en congresos nacionales, apenas tienen presencia en los congresos europeos. Así mismo, puede notarse que el congreso mundial apenas ha suscitado interés en nuestra Comunidad. También se observa que, exceptuando un estudio en que participaron 5 hospitales de la provincia de Pontevedra (13), las comunicaciones se realizan por cada uno de los centros individualmente. En la tabla 2 se enumeran los hospitales y los campos de investigación considerados como consolidados dentro de ellos, atendiendo al criterio de que hayan remitido comunicaciones sobre el mismo tema a 2 o más congresos. Dicho criterio quizá sea considerado poco exigente para suponer consolidado un campo de investigación pero refleja, a nuestro entender, una continuidad de trabajo de al menos varios años sobre dicho campo. Como puede verse son ya 9 los hospitales gallegos que cumplen este criterio, existiendo incluso en varios de los hospitales mayores equipos diferentes dedicados a la misma parcela investigadora.

\section{DISCUSIÓN}

En una reflexión reciente que constata el creciente descenso de comunicaciones sometidas a evaluación en los congresos nacionales de Hepatología, se plantea la existencia de una crisis de investigación en nuestros hospitales. Como factores contribuyentes se apuntan el envejecimiento de las plantillas, la mayor presión asistencial, la poca interrelación entre investigación básica y clínica y la devaluación del curriculum científico14. En otro artículo sobre el futuro de la investigación en 
TABLA I

PRODUCCIÓ N CIENTÍFICA CONGRESUAL DE LA M EDICINA INTERNA HOSPITALARIA GALLEGA

\begin{tabular}{|c|c|c|c|c|c|c|c|c|}
\hline & & & Nacionales & & & Europeos & & Mundial \\
\hline & Salamanca-84 & Alicante-94* & Santiago-96 & Granada-98 & Zaragoza-00 & Florencia-99 & Edimburgo-01 & Cancún-00 \\
\hline Juan Canalejo (La Coruña) & 2 & 5 & 20 & 16 & 6 & 5 & & \\
\hline C. Universitario (Santiago) & & 2 & 36 & 13 & 16 & 10 & & \\
\hline Arquitecto M arcide (Ferrol) & 1 & 3 & 13 & 8 & 6 & 1 & 2 & \\
\hline Xeral (Vigo) & & 3 & 13 & 5 & 3 & & & \\
\hline M eixoeiro (Vigo) & & 2 & 1 & 2 & 2 & 1 & 3 & 2 \\
\hline M ontecelo (Pontevedra) & & & 1 & 2 & & & & \\
\hline Xeral-Calde (Lugo) & & 1 & 4 & & & 1 & 1 & \\
\hline Cristal-Piñor (0 urense) & & & 3 & 5 & & & & \\
\hline Otros & & & 7 & 2 & 1 & & 1 & \\
\hline Total Galicia & 3 & 16 & 98 & 53 & 34 & 18 & 7 & 2 \\
\hline Total España & 159 & 611 & 617 & 787 & 626 & 131 & 86 & 44 \\
\hline Galicia/España (\%) & 2 & 3 & 16 & 7 & 5 & 14 & 8 & 5 \\
\hline Total comunicaciones & & 761 & & & & 480 & 365 & 446 \\
\hline
\end{tabular}

*Congreso SEMI + Congreso EAIM .

nuestro país se aboga, en hospitales de gran presión asistencial como los españoles, por una organización investigadora en paralelo con la asistencial, de forma que los investigadores sean los propios responsables de los pacientes. Se propugna, eso sí, una cierta especialización o priorización en los campos de intereses de los clínicos (15). Examinando estas cuestiones en el campo de la investigación clínica de la medicina interna gallega hemos de decir que, en el momento actual, no parece observable este déficit de producción. Por el contrario, en los años examinados y a pesar de la fluctuación en el número de comunicaciones, se observa una creciente incorporación de hospitales a la producción científica. Es así mismo evidente el desarrollo de campos de intereses preferentes por dichos hospitales (Tabla II). En ello indudablemente ha desempeñado un papel fundamental la madurez alcanzada por el sistema MIR en nuestra especialidad. No obstante, y al margen de grandes estudios cooperativos multicéntricos nacionales e interna- cionales puestos en marcha desde hace tiempo, o los más recientes bajo el auspicio de la SEMI (insuficiencia cardiaca, enfermedad tromboembólica, riesgo vascular, etc.) con participación de diversos hospitales gallegos, se echa en falta una colaboración interhospitalaria autonómica, de forma semejante a la que se realiza en ciertos campos en otras comunidades (16-19). Sería deseable quizá una participación más activa en los foros internacionales, sobre todo por parte de los hospitales con mayores recursos. Es de notar también, la ausencia de los hospitales comarcales, aunque algunos comienzan a remitir comunicaciones a congresos internacionales. Si se tiene en cuenta que 2 de los hospitales con mayor solera investigadora (Ferrol y Juan Canalejo) ya mandaron comunicaciones en 1984, en el caso de los centros más pequeños quizá haga falta tiempo y apoyo por parte de los demás. En este sentido la creación de grupos multihospitalarios sobre patologías concretas y su relación a través de Internet puede abrir vías para la

\section{TABLA II}

EQ UIPOS HO SPITALARIOS DE M EDICINA INTERNA Y CAM POS DE INVESTIGACIÓ N CO NSO LIDADOS. (Se consideran así cuando han presentado una o más comunicaciones a dos o más congresos).

Juan Canalejo (La Coruña)
Compl. Universitario (Santiago)
Arquitecto M arcide (Ferrol)
Hospital Xeral (Vigo)
M eixoeiro (Vigo)
PO VISA (Vigo)
M ontecelo (Pontevedra)
Xeral-Calde (Lugo)
Cristal-Piñor (O urense)

Cardiovascular, Hepatitis virales, Infecciosas, VIH, Corta estancia. HTA-riesgo cardiovascular, Infecciosas, VIH, Hepatología.

M etabolismo lípidos, Infecciosas, Hepatología.

Síndrome antifosfolípido, Leptina, Infecciosas, VIH, Hepatología.

Cardiovascular, Infecciosas, VIH.

Infecciosas-VIH.

Infecciosas.

Infecciosas, Estudios de supervivencia en infecciones y tumores. Infecciosas-VIH. 
incorporación a la investigación de todos los hospitales. Esto también podría cubrir posibles lagunas en campos tradicionalmente dentro de los intereses de los internistas y que parecen insuficientemente tratados en esta Comunidad (enfermedades sistémicas, metabolismo fosfocálcico, etc.).

Para alcanzar una perspectiva adecuada del logro de los fines de este estudio es preciso ser conscientes de las limitaciones del mismo. En él no se ha pretendido hacer una descripción exhaustiva de la actividad científica de los internistas gallegos. Más bien su objetivo es examinar de algún modo si los internistas de Galicia siguen considerando los congresos de la especialidad en que recibieron su formación primitiva como vehículos válidos para comunicar sus experiencias científicas y describir sus campos preferentes de investigación clínica. De este modo, sólo se han considerado las comunicaciones a congresos nacionales o internacionales de Medicina Interna. Por tanto, es probable que equipos de internistas con campos de intereses específicos y que comunican sus investigaciones en dichos congresos no se vean adecuadamente representados. Un ejemplo concreto. La reciente creación de la Sociedad Gallega para el Estudio del SIDA (SOGAISIDA), adherida a SEISIDA, y que ha celebra-

\section{Bibliografía}

1. Gil Extremera B. Sociedad Española de Medicina Interna. Situación al finalizar el año 2000. Investig Clin 2001; 4: 64-5.

2. Comunicaciones científicas a la XIV Reunión ordinaria de la Sociedad Gallega de Medicina Interna. Galicia Clínica 1997; 69 (1-2):1-60.

3. Rico Irles J. Medicina Interna y Siglo XX en España. Invest Clin 2001; 4: 77-89.

4. Comunicaciones al XVI Congreso Nacional de la Sociedad Española de Medicina Interna. An Med Interna (Madrid) 1994 ( $\mathrm{n}^{\circ}$ extraordinario): $1-45$.

5. Comunicaciones al XXI Congreso Nacional de la Sociedad Española de Medicina Interna-XIIth Congress of European Association of Internal Medicine. An Med Interna (Madrid) 1994; 11 (Supl. 1): 1-187.

6. Comunicaciones al XXII Congreso Nacional de la Sociedad Española de Medicina Interna. An Med Interna (Madrid) 1996; 13 (Supl. 1): 1-209.

7. Comunicaciones al XXIII Congreso Nacional de la Sociedad Española de Medicina Interna. An Med Interna (Madrid) 1998; 15 (Supl. 1): 1-347.

8. Comunicaciones al XXIV Congreso Nacional de la Sociedad Española de Medicina Interna. Rev Clin Esp 2000; 200 (Supl. 1): 1-264.

9. Abstracts to 2rd Congress of the European Federation of Internal Medicine. Eur J Intern Med 1999; 10 (Supl. 1): S23-S214.

10. Abstracts to 3rd Congress of the European Federation of Internal Medicine. Eur J Intern Med 2001; 12 (3): 45-320.

11. Abstracts to 25 th World Congress of Internal Medicine. Medicina Interna Mexico 2000: 16 (Supl.): S1-S176.

12. Díaz-Peromingo JA, García-Suárez F, Iglesias-Gallego M, Sánchez-Leira J, Saborido-Froján J, Albán-Salgado A. Lipoprotein (a) and LDL/HDL index in atrial fibrillation. Abstracts to 3rd Congress of the do ya su $2^{\circ}$ Congreso, está formada mayoritariamente por internistas en el campo del personal facultativo. Es posible que esto limite el número de futuras comunicaciones en este campo a los congresos de la SEMI. Sin embargo, nadie pone en duda que la existencia de esta sociedad constituye un paradigma del enorme vigor de la medicina interna en esta Comunidad. Probablemente lo ideal sería que los internistas pudieran dar a conocer sus investigaciones en los congresos de especialidades pero también se involucraran activamente en los congresos de la SEMI.

En resumen, nuestros resultados constatan que los internistas gallegos están satisfechos de serlo y siguen comunicando sus investigaciones a los congresos de su especialidad. Se ha producido una incorporación progresiva de los diversos hospitales a la producción científica materializada en un número importante y continuado de comunicaciones a congresos nacionales e internacionales y cada uno está desarrollando campos propios y preferentes de investigación. Esto nos lleva a afirmar, en sintonía con lo señalado por el presidente saliente de la SEMI (1), y al menos con referencia a Galicia, el enorme dinamismo de la medicina interna y nos invita a profundizar en la satisfacción de ser y sentirnos internistas.
European Federation of Internal Medicine. Eur J Intern Med 2001; 12 (3): 199-200.

13. Sopeña B, Ocampo A, Morano L, Ojea R, Rancaño M, De la Fuente J, et al. Estudio de la retinitis por citomegalovirus en pacientes con infección por el virus de la inmunodeficiencia humana en la provincia de Pontevedra. An Med Interna (Madrid) 1996; 13 (Supl. 1): 26.

14. Bruguera M. ¿Está en crisis la investigación clínica en los hospitales españoles? Med Clin (Barc) 2001; 117: 625-6.

15. Rodés Teixidor J. El presente y el futuro de la investigación biomédica en España. Rev Clin Esp 2001; 201: 431-5.

16. Kindelán JM, Giménez-Doménech R, Vidal-Verdú E, Madueño J, y Grupo Andaluz para el Estudio de las Enfermedades Infecciosas (GAEI). Prevalencia de mutaciones primarias en el virus de la inmunodeficiencia humana que confieren resistencia a análogos de nucleósidos en pacientes de Andalucía no tratados previamente. Med Clin (Barc) 2000; 115: 423-5.

17. Grupo Castellano-Leonés para el estudio de infección VIH en prisiones. Estudio de casos de infección por el virus de la inmunodeficiencia human en prisiones de Castilla y León. Rev Clin Esp 2001; 201: 249-55.

18. Sociedad Castellano-Leonesa para el estudio de las enfermedades hepáticas. Las lesiones anatomo-patológicas de la hepatitis crónica C como factores predictivos de respuesta mantenida al tratamiento con interferón. An Med Interna (Madrid) 2001; 18: 351-6.

19. Zambrana García JL, Delgado Fernández M, Cruz Caparrós G, Díez García F, Martín Escalante MD, Salas Coronas J. Factores asociados a ingresos inadecuados en un servicio de medicina interna. Med Clin (Barc) 2001; 116: 652-4. 